\title{
Numerical Investigation of Flow and Heat Transfer over a flat plate using Curved Rectangular Winglet Vortex Generators
}

\author{
Abhay P. Asagaonkar *, R.D. Shelke and H. N. Deshpande \\ Department of Mechanical Engineering, PES's MCOE, Savitribai Phule Pune University, Pune, Maharashtra (411007) India \\ Accepted 15 June 2016, Available online 20 June 2016, Special Issue-5 (June 2016)
}

\begin{abstract}
A three dimensional numerical simulation is being carried out to study thermo-hydraulic performance of Curved rectangular winglet vortex generators with elliptical punched hole. The effect of inline and staggered arrangement with curved rectangular winglet vortex generators on a flat plate for heat transfer and. friction factor characteristics is studied numerically and compared with performance of smooth plate. The range of parameters for this study is decided on the basis of present work available. Reynolds number (Re) ranging from 7000 to 30,000, aspect ratio of 1 and angle of attack $(\alpha) 45^{\circ}$ are used for this study. Also, effect of punched elliptical hole is carefully observed. Best thermo-hydraulic performance was obtained for staggered arrangement with increase in Nusselt number (Nu) of about 54\% compared to smooth plate. Results showed that with provision of elliptical hole, increase in Nusselt number (Nu) was observed to be $12.6 \%$ more as compared to vortex generators without holes.
\end{abstract}

Keywords: Vortex generators, Curved rectangular winglet, Heat transfer enhancement, Elliptical hole.

\section{Introduction}

Increasing demands on the performance of heat exchangers used in power systems, automotive industry, electric circuit in electronic chip cooling, air conditioning and refrigerant applications, internal cooling of gas turbine blades and aerospace industry for reasons of compactness, manufacturing cost effectiveness and higher efficiency lead to use of heat transfer enhancement techniques. The main physical mechanisms causing the enhancement of heat transfer is the generation and amplification of sufficiently strong longitudinal vortices which are interacting with the thermal boundary layer. The stratification of the thermal boundary layer near the heated walls is disturbed by these vortices. The convection of warmer fluid perpendicular to the heated wall and the mixing with colder fluid is intensified, and, additionally, further external momentum is transported into the inner boundary layer region. Depending on the specific technical application, flow control devices differ in their geometry, dimensions and integration.

To improve performance, heat exchangers should have a large surface area since the heat transfer takes place on the surface. Various techniques are used to enhance heat transfer. In this study, aluminium is used as the material for the Curved rectangular type winglets. There are two different methods for heat exchange enhancement: active vortex method and

*Corresponding author: Abhay P. Asagaonkar passive vortex method. The active vortex method is used to actively control the secondary flow and pressure drop so as to meet the required heat transfer rates even at the cost of increased pumping power Using longitudinal or latitudinal vortex generators for heat exchange enhancement is known as the passive vortex method. A great deal of research has been done using this method since it is efficient and has low maintenance and operating cost in heat exchangers.

Experimental measurement of the local surface temperature was done using a row of delta winglets (with attack angles of $12.5^{\circ}$ and $25^{\circ}$ ) to generate corotating vortices and a row of rectangular winglets (with an attack angle of $15^{\circ}$ ) to generate counterrotating vortices. Counter-rotating vortices were found to be much more effective than co-rotating vortices in heat transfer augmentation. The local heat transfer enhancement up to $42 \%$ was reported(Edwards et al.(1994)). New winglet type vortex generators namely, punched triangular vortex generators and punched rectangular vortex generators were developed. The Reynolds numbers considered for the channel flow case (based on the hydraulic diameter) ranged from 3288 to 37,817 . Results showed a $23-$ $55 \%$ increase in heat transfer due to the use of vortex generators. Thermal enhancement factor of punched triangular vortex generators was found to be the best and was about 2.92(S. Caliskan et al.(2014).

Three dimensional simulations on flat plate with punched plane and curved winglet type vortex 
generators in inline and staggered arrangements was performed and their results proved that curved winglet type vortex generators have much better heat transfer enhancement and lower flow resistance as compared to corresponding plane winglet vortex generators with provision of punched holes (Guobing et al.(2014). Numerical research showed that compared with plain fin, fin provided with curved delta winglet type vortex generators have average $\mathrm{Nu}$ enhanced by $16.1 \%$ $28.7 \%$, while $\mathrm{f}$ was observed to be increased by $7.6 \%$ 15.2\% (Zhi-Min et al.(2015)). It was reported that curved winglet type vortex generators had it's advantages of heat transfer enhancement and lower flow resistance due to it's streamlined configuration. Also, it was observed that thermal enhancement factor tends to decrease at lower Re and increases at higher values of Re (Russi et al.(2015)).

A modified fin, on which curved rectangular winglet type vortex generators were punched was developed. Their experimental results suggested that when Re was increased from 800 to $3000, N u_{m}$ was found to be increased by $15.4 \%-21.6 \%$ (Bao et al. (2015)). Thermo-hydraulic performance of smooth wavy fin and elliptical tube heat exchangers using different winglet type vortex generators was numerically investigated. It was found that with increasing Re and wavy fin height, decreasing ellipticity ratio, a better heat transfer enhancement was obtained (Babak et al. (2015)). Flow field and heat transfer of fin and tube exchangers provided with different types of longitudinal vortex generators was numerically simulated. Results suggested that $\mathrm{Nu}$ increased up to 205 for fins with longitudinal vortex generators as compared to plain fins. Additionally, rectangular winglet with angle of attack of $45^{\circ}$ obtained best overall performance than other angles of attack in same configuration (Li et al. (2015)).

\section{Gap Analysis}

As we can see from literature survey available, that study on heat transfer investigation with vortex generators by changing vortex generator geometry parameters such as aspect ratio, angle of attack, and type of vortex generator. But there is scope for work to investigate thermo-hydraulic performance by using different arrangements and different types of punched holes, while keeping the other geometrical parameters constant.

\section{Objectives}

From literature review presented above it can be observed that the investigation of heat transfer was being carried out with different angles of attack and by varying dimensions of vortex generators as well as by varying punched hole types. The present work is undertaken to evaluate the performance of the Curved rectangular winglet vortex generator used to enhance the heat transfer rate over a flat plate using inline and staggered configurations. Numerical study is performed for Curved rectangular winglet vortex generators on Aluminium plate of $150 \mathrm{~mm} \times 150 \mathrm{~mm}$ size and $10 \mathrm{~mm}$ thick with angle of attack of $45^{\circ}$ Velocities will be varied such as $1 \mathrm{~m} / \mathrm{s}, 2 \mathrm{~m} / \mathrm{s}, 3 \mathrm{~m} / \mathrm{s}$ and $4 \mathrm{~m} / \mathrm{s}$ and with different heat inputs of $15 \mathrm{~W}, 25 \mathrm{~W}$ and $30 \mathrm{~W}$. The obtained results are to be validated experimentally.

\section{Vortex Generator Geometry}

The geometry of the proposed design is shown in figure. Geometry was created in CREO parametric 2.0 for air flowing through rectangular channel. Longitudinal vortex generator i.e. Curved rectangular winglet is investigated for heat transfer enhancement potential over a flat plate.

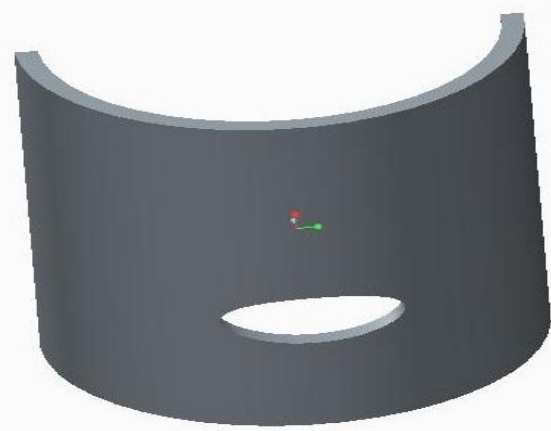

Fig. 1 Vortex Generator Geometry

Vortex generators are made as built-in vortex generators. Aspect ratio was maintained as 1. Height and curvature diameter were kept as $20 \mathrm{~mm}$. Angle of attack is kept fixed at $45^{\circ}$. Elliptical hole of size $8 \mathrm{~mm} x$ $3 \mathrm{~mm}$ is provided at lower half of vortex generator. These vortex generators were arranged in inline as well as staggered configurations.

\section{Grid Independence Study and Boundary conditions}

Grid independence study is carried out over the different numbers of cells. An effort is undertaken to obtain the grid independent results in a rectangular channel. The numbers of grid cells that were used for simulation are 1,65,789, 2,16,918 and 2,74,455. A close agreement is found between the results Obtained for grid cells $2,16,918$ and $2,74,455$. It is observed that the maximum discrepancy in the value of Nusselt number $(\mathrm{Nu})$ for $2,16,918$ and $2,74,455$ is found to be within $0.45 \%$. Hence to save the computational time and cost, grid system of 2,16,918 cells is adopted for this computational model.

Boundary conditions for this study are selected on the basis of data available from literature survey. The Reynolds number ( $\mathrm{Re}$ ) for this study varies from 7000 to 30000 . Constant heat flux condition is used with plate heater providing three step inputs of $15 \mathrm{~W}, 25 \mathrm{~W}$ and $35 \mathrm{~W}$ respectively. Inline and Staggered configurations of vortex generators are being provided on smooth plate. For inlet boundary condition, velocity 
of air is assumed to be uniform and for outlet boundary condition, pressure outlet is assumed to be zero.

\section{Meshing}

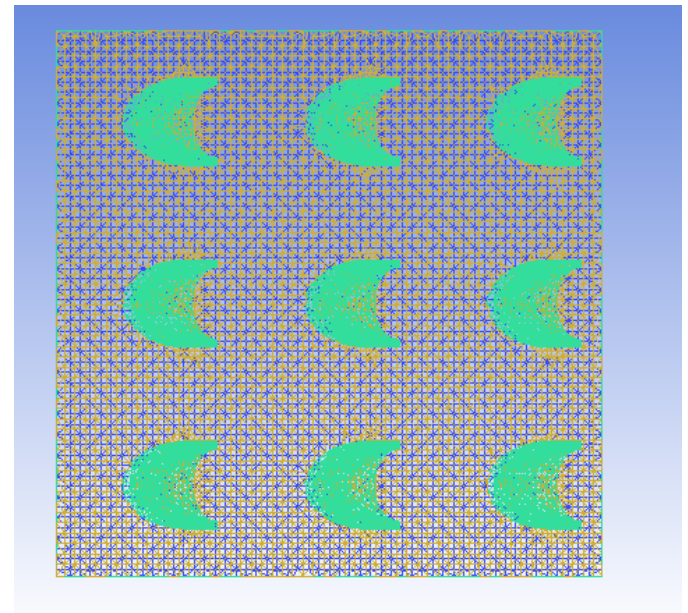

Fig. 2: Computational model of test plate after meshing for inline arrangement

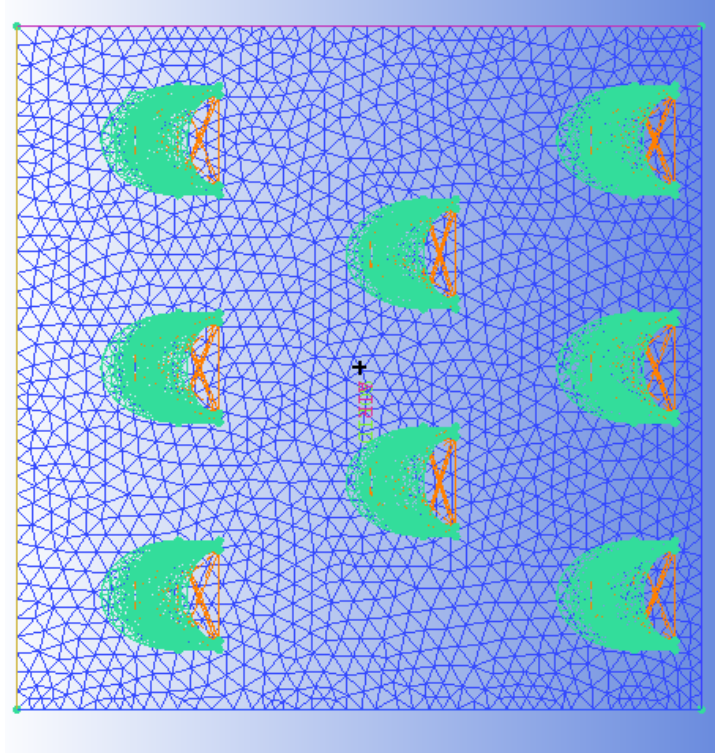

Fig. 3 Computational model of test plate after meshing for staggered arrangement

The computational model of test plate after meshing is shown in fig. 2. The mixed tetra type of mesh is used for this computational model. The mesh density is provided around curved rectangular vortex generators on test plate to get more accurate and reliable results.

\section{Experimental setup and procedure}

The block diagram for experimental setup is as shown in figure 4 . The experimental setup consists of $150 \mathrm{~mm}$ * $150 \mathrm{~mm}^{*} 10 \mathrm{~mm}$ aluminium test plate with vortex generators placed in inline configuration and staggered configuration at a fixed angle of attack of $45^{\circ}$.

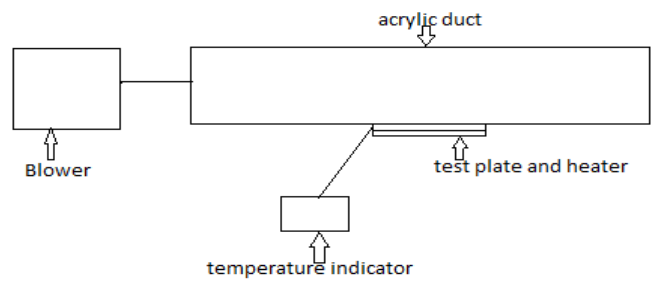

Fig.4: Block diagram for experimental setup

The plate heater is placed exactly below test plate. This test plate assembly with thermocouple as temperature measuring device is placed in acrylic duct of size $2500 \mathrm{~mm} * 150 \mathrm{~mm} * 100 \mathrm{~mm}$. Air blower is connected at inlet section of rectangular channel. Temperature indicator and wattmeter are connected to respective connectors. The proper insulation is provided with insulating tape. The instruments used for experimentation are heater plate of nichrom wire with dimensions same as that of test plate is used, variable speed type blower with rpm range of 1600, Pt-100 Simplex type thermocouples with three core cable, vane probe anemometer with velocity measuring range 0 to $30 \mathrm{~m} / \mathrm{s}$ and wattmeter with capacity to measure 0to750watts is used for the experimentation.

While conducting experiment, constant power input is provided with respective velocity. Temperature of air at inlet and exit of test section and at test plate are recorded at steady state conditions. The data is collected for different specified velocities and power input combinations for smooth plate, inline configuration and staggered configuration.

\section{Data reduction}

Values for air temperatures and plate temperatures are obtained from temperature plots at respective locations. Heat transfer coefficient (h) and Nusselt number $(\mathrm{Nu})$ are calculated from data. Pressure drop $(\Delta \mathrm{P})$ is obtained from pressure plot. These values are used for investigation of various deciding parameters. Following equations are used for evaluation of parameters,

Reynolds number (Re):

$$
R e=\frac{\rho v D h}{\mu}
$$

Heat transfer coefficient $(\mathrm{h})$ :

$$
Q=h A \Delta T
$$

Nusselt number $(\mathrm{Nu})$ :

\section{Results and discussion}

$$
N u=\frac{h D h}{K}
$$

The fig.5, fig.6, and fig.7 shows temperature contours for smooth plate, inline arrangement and staggered arrangement. For smooth plate the temperature profile shows developing boundary layer. With provision of curved rectangular winglet vortex generators, the 
boundary layer is not developed due flow separation and turbulence is caused due to curved rectangular winglet vortex generators.

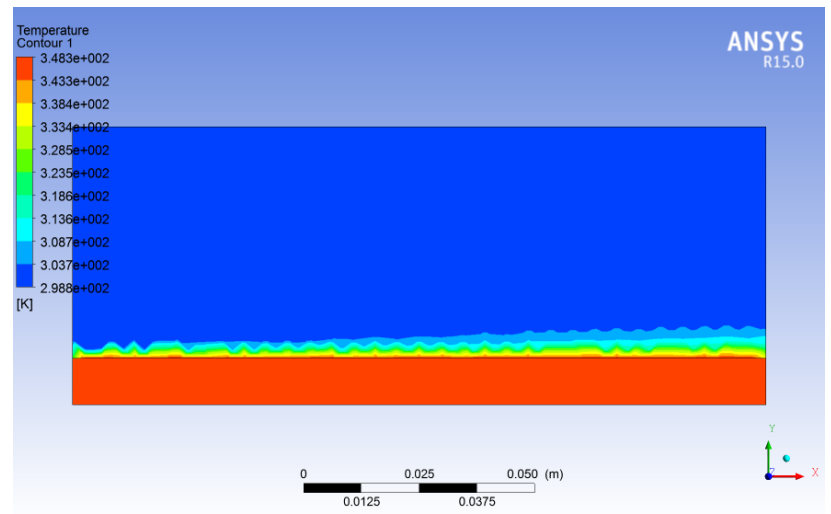

Fig. 5: Temperature contour for smooth plate

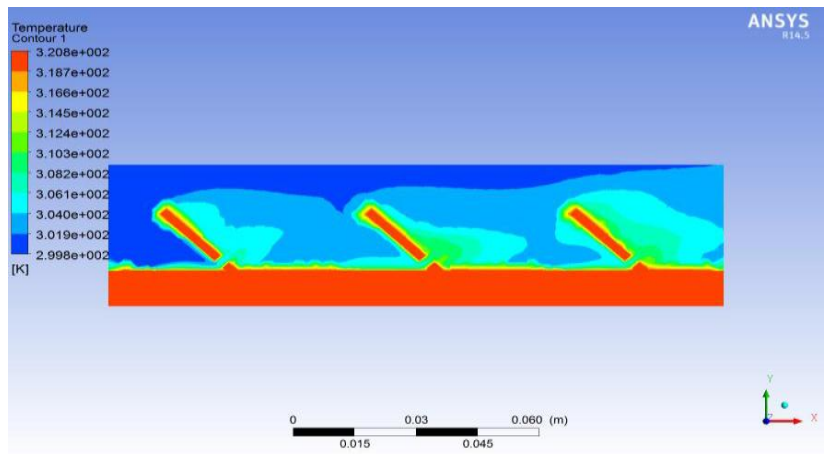

Fig. 6 Temperature contour for inline arrangement

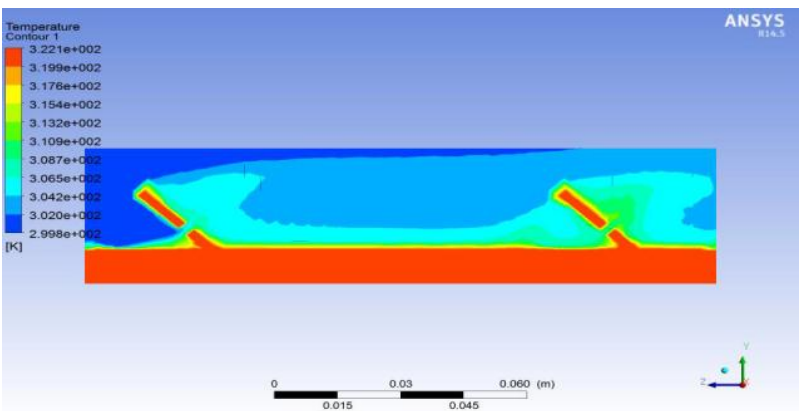

Fig. 7: Temperature contour for staggered arrangement

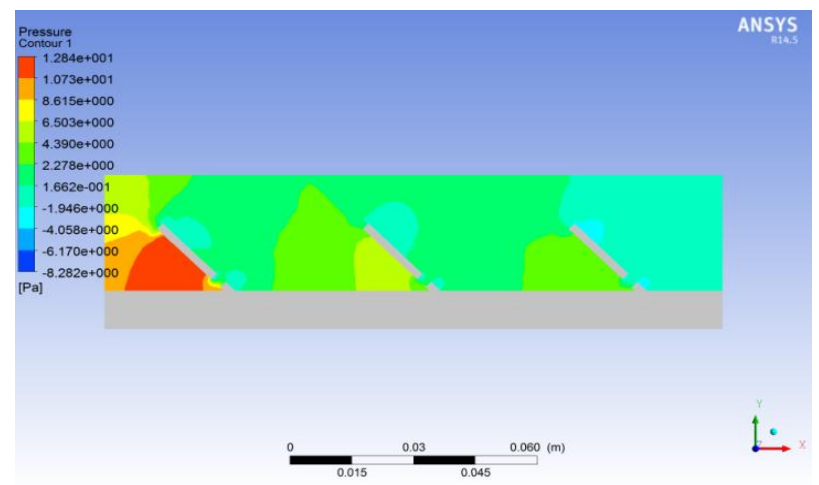

Fig. 9 Pressure contour for inline arrangement

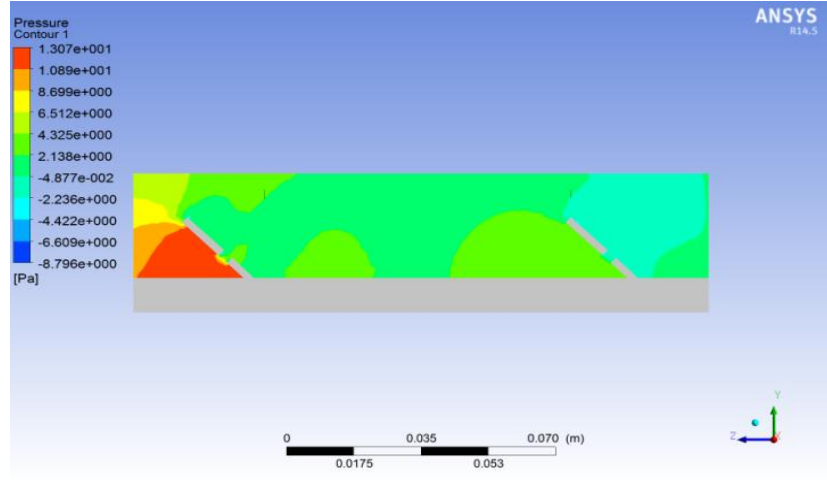

Fig. 10 Pressure contour for staggered arrangement

Figures 8 and 9 presents pressure contours for inline and staggered arrangements. Thus, as Re increases, heat transfer also increases, thereby increasing pressure drop.

Figures 10,11,12 show Comparison of $\mathrm{Nu}$ as a function of Re ranged from 7000 to 30,000 for Smooth plate, inline arrangement and staggered arrangement for different heat inputs. Due to existence of curved rectangular winglet vortex generators with punched elliptical holes, $\mathrm{Nu}$ of inline arrangement and staggered arrangement are higher than that for smooth plate configuration.

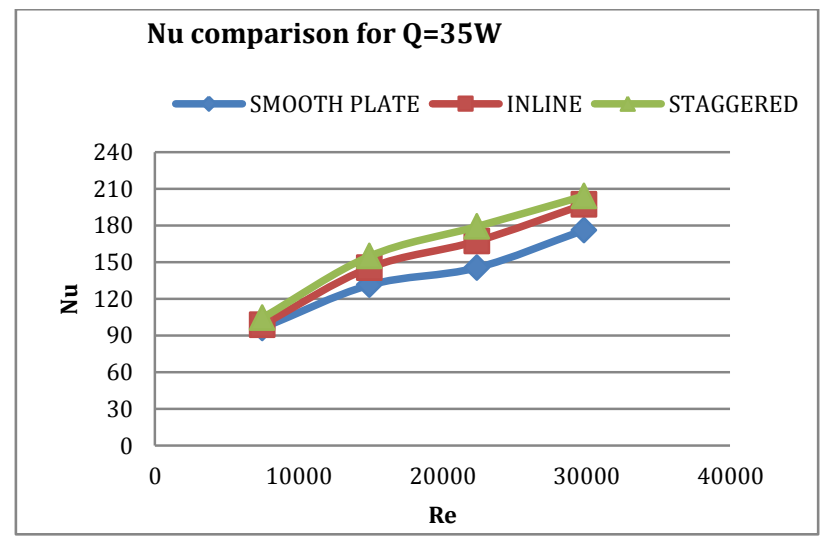

Fig. 10 Comparison of $\mathrm{Nu}$ for Smooth plate, inline arrangement and staggered arrangement for $\mathrm{Q}=15 \mathrm{~W}$

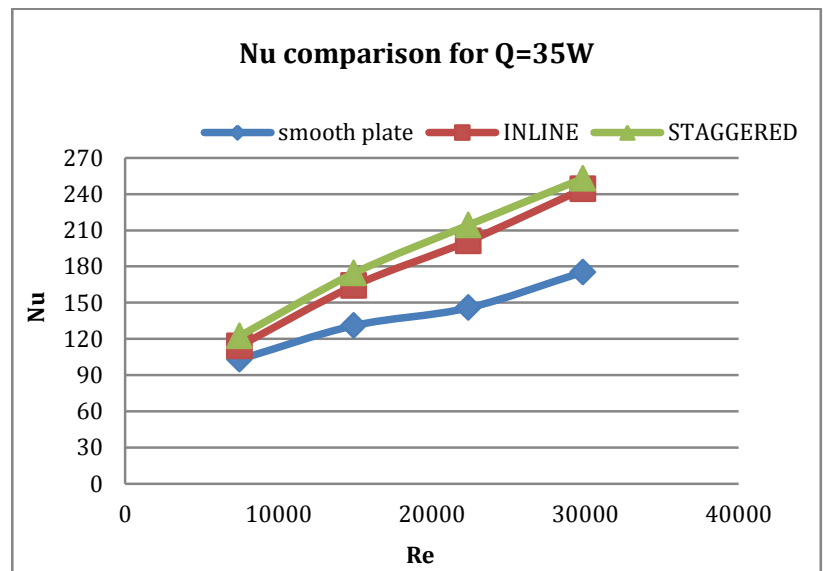

Fig.11:Comparison of $\mathrm{Nu}$ for Smooth plate, inline arrangement and staggered arrangement for $Q=25 \mathrm{~W}$ 


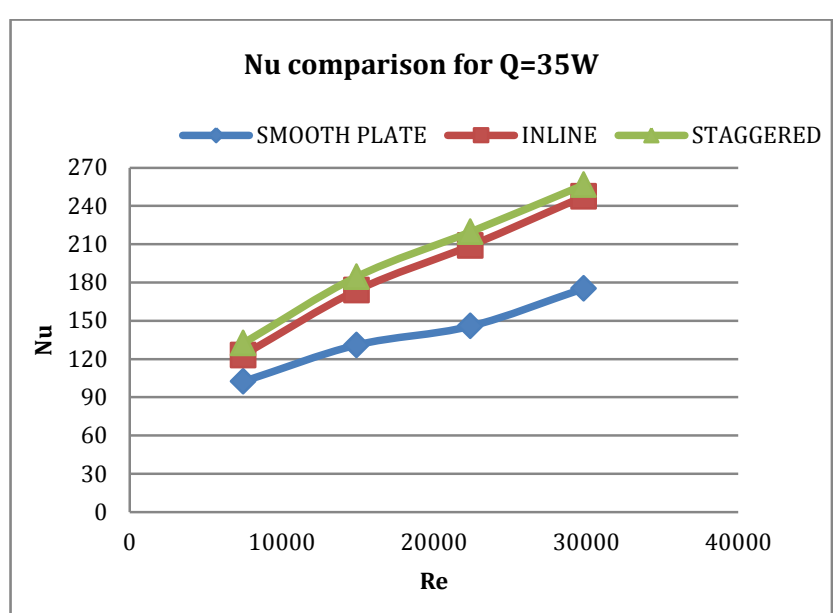

Fig. 12 Comparison of $\mathrm{Nu}$ for Smooth plate, inline arrangement and staggered arrangement for $\mathrm{Q}=35 \mathrm{~W}$

For studied Re range from 7000 to $30,000, \mathrm{Nu}$ is increased by $21 \%-54 \%$. Also, heat transfer enhancement was compared with previous literature available as shown in fig.13. It showed good proximity between results for specified Re range.

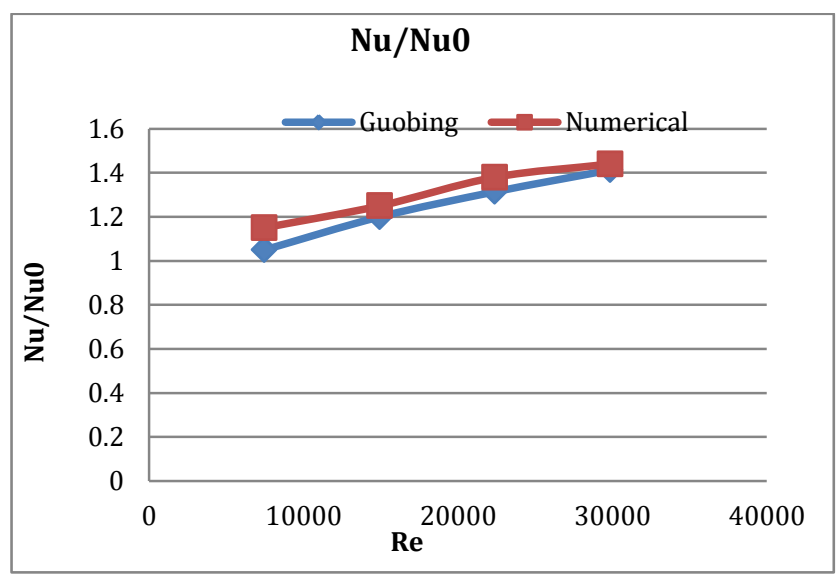

Fig. 13 Validation of numerical results

\section{Conclusions}

Numerical investigation of thermo-hydraulic performance of curved rectangular winglet vortex generators in rectangular channel is being carried out under uniform heat flux condition. Based on the results following conclusions are made:

1) We have clearly seen that as Re goes on increasing, heat transfer coefficient $h$ also increases, thereby increasing $\mathrm{Nu}$.
2) Disturbance in boundary layer is formed due to vortex generators as well as elliptical punched holes, which created higher turbulence due to separated and reattached flows.

3) Best thermo-hydraulic performance was obtained for staggered arrangement with increase in Nusselt number ( $\mathrm{Nu}$ ) of about $54 \%$ compared to smooth plate.

4) With provision of elliptical hole, increase in Nusselt number $(\mathrm{Nu})$ was observed to be $12.6 \%$ more as compared to vortex generators without holes.

\section{References}

Guobing Zhou, Zhizheng Feng,(2014), 'Experimental investigations of heat transfer enhancement by plane and curved delta winglet type vortex generators with punched holes', International Journal of Thermal Sciences,78,pg.2635.

Babak Lotfi , Bengt Sundén, Qiuwang Wang, (2015) , 'An investigation of thermo-hydraulic performance of the smooth wavy fin-and-elliptical tube heat exchangers utilizing new type vortex Generators', Applied Energy Article in press2015.

Bao Gong , Liang-Bi Wang , Zhi-Min Lin , (2015), 'Heat transfer characteristics of a circular tube bank fin heat exchanger with fins punched rectangular vortex generators in the wake regions of the tubes',Applied Thermal Engineering,75, 224-338.

S. Caliskan, (2014), 'Experimental investigation of heat transfer in a channel with new winglet-type vortex generators', International Journal of Heat and Mass Transfer,78604-614.

Zhi-Min Lin , Cai-Ping Liu, Mei Lin , Liang-Bi Wang , (2015), 'Numerical study of flow and heat transfer enhancement of circular tube bank fin heat exchanger with delta-winglet vortex generators', Applied Thermal Engineering,88, 198210

Edwards, F. J., and Alker, C. J. R.,(1994),"The Improvement of Forced Convection Surface Heat Transfer Using Surface Protrusions in the Form of (a) Cubes and (b) Vortex Generators", Fifth Int. Heat Transfer Conf. JSME Tokyo 2, 2244-2248.

Li Li, Xiaoze Du, Yuwen Chang,2015" Numerical investigation of heat transfer of fin and tube heat exchanger with curved winglet type vortex generators", International Journal of Thermal Sciences, pg 85-96.

Russi, Sunil Dhingara,(2015) ,Numerical investigation of heat transfer enhancement by plain and curved winglet type vortex generators",IJSRD,Vol.2,Issue4.

Fiebig, M., Valencia, A. and Mitra, N.,(1993),"Wing-Type Vortex Generators for Fin-and Tube Heat Exchangers", Experimental Thermal and Fluid Science 7, 287-295 